\title{
Statistical Analysis of Correlated Relative Risks
}

\author{
Rickey E, Carter ${ }^{1,2}$, Xuyang Zhang ${ }^{3}$, Robert F. Woolson ${ }^{1}$ \\ and Christian C. Apfel ${ }^{4}$ \\ ${ }^{1}$ Medical University of South Carolina, ${ }^{2}$ Mayo Clinic, ${ }^{3}$ Nationwide Insurance \\ and ${ }^{4}$ University of San Francisco
}

\begin{abstract}
Much of the statistical literature regarding categorical data focuses on the odds ratio, yet in many epidemiological and clinical trial settings, the relative risk is the quantity of interest. Recently, Spiegelman and Hertzmark illustrated modeling and SAS programming for modeling relative risk in contrast to the logistic model's odds ratio. The focus of their work is on a single relative risk, i.e., for one binary response variable. Herein, we outline two methods for estimating relative risks for two correlated binary outcomes. The first method is weighted least squares estimation for categorical data modeling. The second method is based on generalized estimating equations. The two methods are readily implemented using common statistical packages, such as SAS. The methods are illustrated using clinical trial data examining the relative risks of nausea and vomiting for two different drugs commonly used to provide general anesthesia.
\end{abstract}

Key words: Correlated binary data, generalized estimating equations, odds ratio, postoperative nausea and vomiting, relative risk, weighted least squares.

\section{Introduction}

Statistical methods are well established for a variety of research designs. Often, the research study will utilize a binary endpoint (e.g., a 'success' or 'failure'). Cornfield (Cornfield 1951) discussed two measures of association for comparing binomial proportions across groups, and these measures are applied broadly in research. To illustrate the methods, consider a standard $2 \times 2$ contingency table in which the binary outcome is presented as the columns and a two group treatment (or exposure) is presented as the rows. Denote $\pi_{i}$ as $P$ (Success $\mid$ Treatment $i$ ) and $o_{i}=\pi_{i} /\left(1-\pi_{i}\right)$ as the odds of a successful outcome for Treatment $i, i=1,2$. To quantify the treatment differences in $\pi_{i}$ or $o_{i}$, either the relative risk or odds ratio could be used. The relative risk is defined as $R R=\pi_{1} / \pi_{2}$, i.e. the ratio of the two success probabilities, and measures the proportional increase (or decrease) 
in the probability of a 'success' in Treatment 1 when compared to Treatment 2. Cornfield also proposed the odds ratio $\left(o_{1} / o_{2}\right)$ as a relative measure of association that measures the proportional increase (or decrease) in the odds of a 'success' in Treatment 1 relative to Treatment 2. Mathematically, these quantities are related as follows:

$$
O R=R R\left(\frac{1-\pi_{2}}{1-\pi_{1}}\right)
$$

In clinical trials and prospective epidemiological studies the relative risk is typically of principal interest. Yet, the logistic regression model, based on the log odds (i.e., logit) transformation, is often the first model fit to "approximate" the relative risk via the odds ratio. Recently, Spiegelman and Hertzmark (Spiegelman and Hertzmark 2005) observed that computational advances no longer necessitate this approximation of relative risk by the odds ratio. Stated simply, if interest is in the relative risk, then model the relative risk, not the odds ratio. Universal application of a logistic regression model for binary outcomes is not required for every prospective study data analysis. Other links, in the context of generalized linear models, can be used to model the success probability for a binary outcome. This leads to direct calculation of the relative risk, and with additional calculations a variance estimate and a corresponding confidence interval for the relative risk are produced.

Spiegelman and Hertzmark describe log-linear models for estimating relative risk. The methods of Spiegelman and Hertzmark can be implemented using standard GLM-fitting software (e.g., PROC GENMOD in SAS); however, the approach for estimating relative risk for two (or more) correlated binary outcomes requires extensions. The subject of this paper is to describe these generalizations for both weighted least squares (WLS) and generalized estimating equation (GEE) approaches.

We describe two methods for estimating the relative risk of correlated binary endpoints. The first method, a WLS approach, is an application of the Grizzle et al. (Grizzle et al. 1969) approach to modeling categorical data. The second method utilizes GEE to allow for the adjustment of continuous and/or categorical covariates in the model ( $\mathrm{Lu}$ et al. 2001). Both methods can be implemented with standard statistical software packages. The methods are illustrated using an international, multi-center study evaluating the efficacy of propofol as a therapy to prevent post-operative vomiting and nausea (Apfel et al. 2003, Apfel et al. 2004).

The paper is organized as follows. The statistical methods for the WLS and GEE approaches are outlined first. Next, the methods are applied, and interpreted, for a clinical trial. 


\section{Methods}

\subsection{Weighted least squares approach}

Grizzle et al. (Grizzle et al. 1969) developed a linear models approach for categorical data, and it is applicable to this setting. In the presence of only one covariate (e.g., treatment group), two correlated binary outcome responses can be presented as a $2 \times 2 \times 2$ three-way contingency table. Table 1 illustrates a setting in which treatment group is one dimension, while the remaining two dimensions are the paired binomial responses. Thus, the following multinomial probability vectors, which are based on the contingency table presented as Table 1 , are defined for the treatment and control groups:

$$
\begin{aligned}
& \pi_{t}=\left[\begin{array}{llll}
\pi_{t 11} & \pi_{t 12} & \pi_{t 21} & \pi_{t 22}
\end{array}\right]^{\prime} ; \\
& \pi_{c}=\left[\begin{array}{llll}
\pi_{c 11} & \pi_{c 12} & \pi_{c 21} & \pi_{c 22}
\end{array}\right]^{\prime} ; \\
& \text { and } \quad \pi=\left[\begin{array}{ll}
\pi_{t}^{\prime} & \pi_{c}^{\prime}
\end{array}\right]^{\prime} .
\end{aligned}
$$

The variance-covariance matrix for $\pi$ follows the usual form for two independent multinomials, namely,

$$
\begin{aligned}
V(\pi) & =\left[\begin{array}{cc}
V_{t}\left(\pi_{t}\right) & 0 \\
0 & V_{c}\left(\pi_{c}\right)
\end{array}\right], \text { where } \\
V_{x}\left(\pi_{x}\right) & =\frac{1}{n_{x .}}\left[\operatorname{Diag}\left(\pi_{x}\right)-\pi_{x} * \pi_{x}^{\prime}\right]_{4 \times 4} \\
& \text { for } x \in(t, c) .
\end{aligned}
$$

\begin{tabular}{|c|c|c|c|c|}
\hline \multicolumn{5}{|c|}{ Cell Counts (Cell Probability) } \\
\hline & Outcome 1 & + & - & Total \\
\hline \multicolumn{5}{|l|}{ Treatment Group } \\
\hline & + & $n_{t 11}\left(\pi_{t 11}\right)$ & $n_{t 12}\left(\pi_{t 12}\right)$ & $n_{t 1 \cdot}\left(\pi_{t 1 \cdot}\right)$ \\
\hline & - & $n_{t 21}\left(\pi_{t 21}\right)$ & $n_{t 22}\left(\pi_{t 22}\right)$ & \\
\hline & Total & $n_{t \cdot 1}\left(\pi_{t \cdot 1}\right)$ & & $n_{t .}$ \\
\hline \multirow[t]{3}{*}{ Control Group } & + & $n_{c 11}\left(\pi_{c 11}\right)$ & $n_{c 12}\left(\pi_{c 12}\right)$ & $n_{c 1} \cdot\left(\pi_{c 1} \cdot\right)$ \\
\hline & - & $n_{c 21}\left(\pi_{c 21}\right)$ & $n_{c 22}\left(\pi_{c 22}\right)$ & \\
\hline & Total & $n_{c \cdot 1}\left(\pi_{c \cdot 1}\right)$ & & $n_{c .}$ \\
\hline
\end{tabular}

Table 1: Frequency distribution and cell probabilities for two paired binomial responses measured on two treatments.

Since the interests of the study are in the two marginal outcome probabilities for each treatment group and the comparison between groups, the response functions of interest are $\left.f_{t}=\left[\log \left(\pi_{t 11}+\pi_{t 12}\right)\right], \quad \log \left(\pi_{t 11}+\pi_{t 21}\right)\right]$ l and 
$\left.f_{c}=\left[\log \left(\pi_{c 11}+\pi_{c 12}\right)\right], \quad \log \left(\pi_{c 11}+\pi_{c 21}\right)\right]$ / for the treatment and control groups, respectively. Therefore, the response vector is $f(\pi)=\left[\begin{array}{ll}f_{t}^{\prime} & f_{c^{\prime}}\end{array}\right]$ '

When data are observed, the estimated cell probabilities are $p_{x i j}=\hat{\pi}_{x i j}=$ $n_{x i j} / n_{x . .}$, where $x$ indicates treatment $(x=t)$ or control $(x=c)$. This yields the estimated vector of probabilities $p=\left[p_{t}^{\prime} p_{c}^{\prime}\right]$, where $p_{t}^{\prime}=\left[n_{t 11} / n_{t . .}, \ldots, n_{t 22} / n_{t . .}\right]$ and $p_{c}^{\prime}=\left[n_{c 11} / n_{c . .}, \ldots, n_{c 22} / n_{c . .}\right]$. An estimated variance-covariance matrix of $f(p)$ can be obtained by the delta method as $S=H * V(p) * H$, where $V(p)$ is the sample estimate of $V(\pi)$ and $H$ is as follows:

$$
\begin{aligned}
& H=\left.\frac{\partial f}{\partial \pi}\right|_{\pi=p} \\
& =\left[\begin{array}{ccccccccc}
\frac{n_{t . .}}{n_{t 11}+n_{t 12}} & \frac{n_{t . .}}{n_{t 11}+n_{t 12}} & 0 & 0 & 0 & 0 & 0 & 0 \\
\frac{n_{t .}}{n_{t 11}+n_{t 21}} & 0 & \frac{n_{t . .}}{n_{t 11}+n_{t 21}} & 0 & 0 & 0 & 0 & 0 \\
0 & 0 & 0 & 0 & \frac{n_{c . .}}{n_{c 11}+n_{c 12}} & \frac{n_{c . .}}{n_{c 11}+n_{c 12}} & 0 & 0 \\
0 & 0 & 0 & 0 & \frac{n_{c . .}}{n_{c 11}+n_{c 21}} & 0 & \frac{n_{c . .}}{n_{c 11}+n_{c 21}} & 0
\end{array}\right] .
\end{aligned}
$$

Note that although the two response functions from the same group are correlated (both are functions of $n_{t 11}$ for the treatment group and $n_{c 11}$ for the placebo group), the sample variance-covariance matrix, $S$, is of full rank and a model containing up to four parameters is estimable.

One can see that the difference in the first and third elements in $f(\pi)$ defines the log relative risk for the first binary outcome when the treatment is compared to the control. Similarly, the difference in the second and fourth entries in $f(\pi)$ defines the log relative risk for the second binary outcome. In order to find the WLS estimate for the log relative risk using the four estimable parameters $\left(\beta=\left[\beta_{1}, \beta_{2}, \beta_{3}, \beta_{4}\right] \prime\right)$ in the saturated model, we utilize the following model matrix such that $f(\pi)=X \beta$ :

$$
X=\left[\begin{array}{cccc}
1 & 1 & 1 & 0 \\
1 & -1 & 0 & 1 \\
1 & 1 & -1 & 0 \\
1 & -1 & 0 & -1
\end{array}\right]
$$

The model, parameterized as such, allows for the estimation of the marginal $\log$ relative risks for each of the two binary outcomes while accounting for the dependence of the two outcomes. The WLS estimates of $\beta$ are: $\hat{\beta}=\left(X^{\prime} S^{-1} X\right)^{-1} X^{\prime}$ $S^{-1} f(p)$ and the estimated variance-covariance matrix of $\widehat{\beta}$ is $\operatorname{Cov}(\hat{\beta})=\left(X^{\prime} S^{-1} X\right)^{-1}$.

The difference of the first and third rows in $f(\pi)$ is of clinical interest and is the $\log$ relative risk for the first outcome. In terms of the linear predictor defined by the model matrix, this difference is simply $2 \beta_{3}$. Similarly, the log relative risk for the second binary outcome is $2 \beta_{4}$. The standard error, Wald tests, and confidence intervals of such scaled values may be calculated using the estimated 
variance-covariance matrix of $\widehat{\beta}$. In addition, the estimate of the log relative risk may be exponentiated to produce an estimate for relative risk. A $95 \%$ confidence interval for the relative risk for the first binary outcome is

$$
\exp \left[2 \hat{\beta}_{3} \pm 1.96 \sqrt{2^{2} \operatorname{Cov}(\hat{\beta})_{3,3}}\right]=\exp \left[2\left(\hat{\beta}_{3} \pm 1.96 \sqrt{\operatorname{Cov}(\hat{\beta})_{3,3}}\right)\right]
$$

The difference in estimated marginal relative risks also may be of clinical interest. Testing this difference against zero would be testing whether or not the treatment is equally effective (or ineffective) for the two binary outcomes at significance level $\alpha$. The $95 \%$ confidence interval for this difference is

$$
\exp \left[2\left(\hat{\beta}_{3}-\hat{\beta}_{4} \pm 1.96 \sqrt{\left.\operatorname{Cov}(\hat{\beta})_{3,3}+\operatorname{Cov}(\hat{\beta})_{4,4}-2 \operatorname{Cov}(\hat{\beta})_{3,4}\right)}\right],\right.
$$

which is centered around the point estimate of $\exp \left[2\left(\hat{\beta}_{3}-\hat{\beta}_{4}\right)\right]$. The correlation between $\widehat{\beta_{3}}$ and $\widehat{\beta_{4}}$ is estimated as

$$
\frac{\operatorname{Cov}(\hat{\beta})_{3,4}}{\sqrt{\operatorname{Cov}(\hat{\beta})_{3,3} \operatorname{Cov}(\hat{\beta})_{4,4}}} .
$$

Hence, apart from the occasional zero cell count, this approach to modeling these correlated log relative risks is relatively straightforward.

\subsection{Generalized estimating equation (GEE) approach}

While the WLS approach is appropriate for randomized clinical trials, often there is a need to adjust the analysis for design variables such as clinical site or additional baseline variables, some of which may not be categorical. In this instance, a GEE approach is more flexible and can be applied ( $\mathrm{Lu}$ et al. 2001). To formulate the model, suppose that $i^{\text {th }}$ subject has a bivariate outcome vector $Y_{i}=\left[\begin{array}{ll}Y_{i 1} & Y_{i 2}\end{array}\right]$, for $i=1,2, \ldots, N$. Let $E\left[Y_{i j} \mid X_{i j}\right]=P\left(Y_{i j}=1 \mid X_{i j}\right)=P_{i j}$ for $j=1,2$ be the probability of observing a positive response on the first binary outcome $(J=1)$ or on the second $(J=2)$ for the $i^{\text {th }}$ subject on the $j^{\text {th }}$ outcome. When a logarithm link function is used, the log-linear function of $P_{i j}$ in $X_{i j}$ is $\log \left(P_{i j}\right)=X_{i j} \beta$, where $\beta$ is a $p \times 1$ regression coefficient vector. Thus, $P_{i}=$ $\left[P_{i 1}, P_{i 2}\right] \prime=\left[\exp \left(X_{i 1} \beta\right), \exp \left(X_{i 2} \beta\right)\right]$.

Similar to the WLS approach, the model matrix configuration is of importance. The coding of values of the model matrix is as follows: the first column is the intercept with a value of 1 for all rows; the second column is the indicator for which of the two binary outcomes the row is referencing; the third column is the group identity ( 1 if the subject is in the treatment group and 0 if the subject is in the control group); the fourth column codes the interaction between 
outcome indicator and treatment. This model matrix is for when the interaction between binary outcomes and treatment (i.e., differential treatment effect on the two binary outcomes) is of interest, and can be illustrated as

$$
X_{i}=\left[\begin{array}{l}
X_{i 1} \\
X_{i 2}
\end{array}\right]=\left[\begin{array}{llll}
1 & 1 & 1 & 1 \\
1 & 0 & 1 & 0
\end{array}\right]
$$

if the $i^{\text {th }}$ subject is on treatment and

$$
X_{i}=\left[\begin{array}{l}
X_{i 1} \\
X_{i 2}
\end{array}\right]=\left[\begin{array}{llll}
1 & 1 & 0 & 0 \\
1 & 0 & 0 & 0
\end{array}\right]
$$

if the $i^{t h}$ subject is on control.

The parameter vector consists of four elements, $\beta=\left[\beta_{1}, \beta_{2}, \beta_{3}, \beta_{4}\right] \prime$, as before. $\beta_{4}$ measures the treatment effect on the first outcome beyond that for the second outcome. If there is no differential effect on the two outcomes, then the interaction term may be deleted and the parameter $\beta_{3}$ would be the common log relative risk of the treatment relative to the control. The solutions, $\widehat{\beta}$ and $\widehat{\alpha}$, the correlation of the binary outcomes, are obtained iteratively as described elsewhere (Zeger and Liang 1986). This method can be implemented using SAS PROC GENMOD with a logarithm link function and binomial distribution specification in the model statement. The correlation of the binary outcomes is specified with the REPEATED statement. Note that this design accommodates additional covariates in the design matrix and produces "adjusted" relative risks in the same manner as a multiple logistic regression produces adjusted odd ratios.

\section{Example}

Annually, over 75 million surgical patients world wide are administered anesthesia, and up to one third of these patients will develop some combination of nausea and vomiting if left untreated during post-operative recovery (Apfel et al. 2004). Clinically, nausea is an unpleasant sensation in the abdomen and may provide significant discomfort to the patient. However, vomiting poses a greater medical risk in that the forcible expulsion of stomach contents through the mouth may cause damage to surgical site. These two adverse effects are commonly considered paired (e.g., occurrence of nausea and vomiting), and in fact, nausea often times culminates in vomiting. As such, the assessment of adjuvant therapies to minimize the incidence of nausea and/or vomiting in patients after general anesthesia is of clinical importance. Correspondingly, numerous studies have been conducted to date, and one recent trial provides the motivation for this research. 
Apfel et al. (Apfel et al. 2003) conducted IMPACT (an International Multicenter Protocol to assess the single and combined benefits of Antiemetic interventions in a controlled Clinical Trial of a $2 \times 2 \times 2 \times 2 \times 2 \times 2$ factorial design), for the prevention of postoperative nausea and vomiting. For the purpose of this illustration, we consider two of the randomized treatments, namely propofol versus volatile anesthetics. The volatile anesthetics group serves as the control group. Initially, the data were analyzed using the odds ratio (Apfel et al. 2004); however, IMPACT is a prospective clinical trial design, and the effect of propofol on the symptoms is more appropriately expressed in terms of relative risk. The research question is whether or not propofol is effective in controlling postoperative vomiting and nausea and, when it is effective, whether it is equally effective for controlling vomiting and nausea.

To answer these questions, IMPACT evaluated the incidence of nausea and vomiting on 5,159 participants. Of these, 3,426 were randomized into the propofol treatment group and the remaining 1,733 were randomized into the control group to whom only the volatile anesthetic was provided. The outcome data for this study are presented as Table 2. The incidence of nausea appears higher than that of vomiting on both treatments and similarly, the incidence of both nausea and vomiting appear lower on the propofol group than on the control group.

Table 2: Realization of IMPACT study presented in the Context of Table 1

\begin{tabular}{lcccc}
\hline \multicolumn{3}{l}{ Cell Counts (Estimated Cell Probability) } & & \\
Treatment Group & Vomiting & + & - & Nausea \\
\hline & + & $358(0.1045)$ & $23(0.0067)$ & $381(0.1112)$ \\
& - & $677(0.1976)$ & $2368(0.6912)$ & \\
\cline { 2 - 5 } & Total & $1035(0.3021)$ & & 3426 \\
\hline Control Group & + & $223(0.1287)$ & $13(0.0075)$ & $236(0.1362)$ \\
& - & $426(0.2458)$ & $1071(0.6180)$ & \\
\cline { 2 - 5 } & Total & $649(0.3745)$ & & 1733 \\
\hline
\end{tabular}

The estimation for the relative risks of nausea and vomiting using the WLS approach was conducted using PROC CATMOD in SAS (SAS Institute Inc. 2001). When WLS is applied to the data from IMPACT, the estimates in Table 3 are obtained. Using these WLS estimates and testing procedures, it was found that propofol had a significant effect in controlling vomiting and nausea. The relative risk for these two symptoms are, respectively, 0.82 (95\% C.I. from 0.70 to 0.95 ) and 0.81 (95\% C.I. from 0.75 to 0.87 ) with the control group's risks as the references. Using the $95 \%$ confidence interval for the ratio of relative risks 
$(0.89,1.16)$ suggests that propofol is equally effective for the two symptoms since the $95 \%$ confidence interval includes 1 and is quite narrow.

Table 3: Relative risks (RR) and confidence intervals estimated for the IMPACT clinical trial examining the effects of propofol relative to volatile anesthetic for controlling post operative nausea and vomiting.

\begin{tabular}{|c|c|c|c|c|}
\hline & \multicolumn{2}{|c|}{ Weighted Least Squares } & \multicolumn{2}{|c|}{ Generalized Estimating Equations } \\
\hline & $\mathrm{RR}$ & $95 \%$ CI & $\mathrm{RR}$ & $95 \%$ CI \\
\hline Vomiting & 0.82 & $(0.70,0.95)$ & 0.82 & $(0.70,0.95)$ \\
\hline Nausea & 0.81 & $(0.75,0.87)$ & 0.81 & $(0.75,0.87)$ \\
\hline $\begin{array}{l}\text { Ratio of relative risks } \\
\text { for vomiting and nausea }\end{array}$ & 1.01 & $(0.89,1.16)$ & 1.01 & $(0.89,1.16)$ \\
\hline $\begin{array}{l}\text { Common relative risk } \\
\text { for vomiting and/or nausea } \\
\text { with propofol vs } \\
\text { volatile anesthetics }\end{array}$ & & & 0.81 & $(0.75,0.87)$ \\
\hline
\end{tabular}

To implement the GEE method, the data format was restructured/organized such that each subject had two rows of data, one for each symptom. Values for the subject on the design matrix with dimension of $2 \times 4$ were determined according to his/her group identity (propofol or control; refer to the design matrix part of Section 2.2). Thus, the first row for each subject coded the person's conditions related to his/her response on vomiting and the second row coded that for the nausea response. $Y_{i}=\left[\begin{array}{ll}Y_{i 1}, & Y_{i 2}\end{array}\right]$, where $Y_{i 1}=1$ (or 0$)$ for a positive (or negative) response on vomiting and $Y_{i 2}=1$ (or 0 ) for a positive (or negative) response on nausea. A positive response is defined as the observation of the symptom within 24 hours post surgery.

PROC GENMOD in SAS was used to obtain the estimates of $\beta$. When these estimates were exponentiated, estimates for relative risk and its confidence intervals were obtained. The results are listed in Table 3 . The data supports the hypothesis that propofol does not provide differential effects for either nausea or vomiting $(\mathrm{p}=0.86)$, and the estimated relative risks and $95 \%$ confidence intervals for propofol's effect on nausea or vomiting are identical to that obtained from the WLS method when the interaction of treatment and symptom are included in the model. Eliminating the interaction term from the model yields an estimate for the pooled (or common) relative risk of 0.81 and corresponding $95 \%$ confidence interval of $(0.74,0.87)$. The results are concordant for the two methods in this example; in other settings, there may be good reason to use one method over the other including the desire to incorporate continuous covariables. 


\section{Discussion}

Estimation of relative risk in lieu of the more common odds ratio should be considered when the data arise from a prospective study (Spiegelman and Hertzmark 2005). In this paper we developed methods for modeling correlated relative risks, noting that these techniques are illustrations of Grizzle et al. (Grizzle et al. 1969) linear model approach to categorical data and GEE method by Liang and Zeger (Zeger and Liang 1986). These methods can be implemented using common statistical software, or may be implemented with software supporting matrix operations. The syntax for the motivating example using SAS PROC GENMOD, SAS PROC CATMOD, and SAS IML, are available upon request from the first author.

The WLS approach directly utilizes the analytical expressions for the variance/covariance of the binary outcomes. Thus, in situations where only one dichotomous covariable is of interest (e.g., treatment), WLS is preferred. The GEE approach illustrated naturally extends to situations with more than two correlated binary outcomes, and is preferable in instances when adjustment for additional covariables is necessary. Convergence difficulties in the bernoulli likelihood, using either WLS or GEE, may occur when the success probabilities approach one (Wacholder 1986, Zou 2004, Carter et al. 2005, Spiegelman and Hertzmark 2005, Blizzard and Hosmer 2006). Utilizing the Poisson likelihood with robust variance estimator improves convergence in the univariate setting (Zou 2004, Carter et al. 2005) and naturally extends to the multivariate setting when using GEE as outlined above.

Many statisticians prefer the canonical link (i.e., logit transformation) when analyzing binary outcomes. In the case of rare diseases, the odds ratio will approximate the relative risk, but in clinical trials with non-rare outcomes, the relative risk will be overestimated by the odds ratio. Zhang and Yu (Zhang and $\mathrm{Yu}$ 1998) propose a technique for estimating RR based on the mathematical relationship of $\mathrm{OR}$ with $\mathrm{RR}$. This approach utilizes the predicted probabilities obtained from a logistic regression model, but in the presence of multiple covariates, this approach does not produce adjusted estimates of relative risk due to the need to fully specify the values for the covariate vector. Moreover, this approach produces inconsistent estimates for relative risk and invalid confidence intervals (Zou 2004).

The motivating example originated through collaboration with the principal investigator of IMPACT. The example of nausea and vomiting expresses the notion of correlated binary endpoints well. If additional covariates are considered, then further GEE modeling could be considered. This example presents an examination of adverse effects patients can encounter with anesthetic therapies. The 
statistical modeling illustrates the ease with which correlated relative risks can be estimated directly and compared in clinical trials and other prospective studies.

\section{Acknowledgements}

The support for the development of the statistical methodology partially comes from the National Institutes of Health grants RR01070 and DA013727. The clinical study "IMPACT" was supported by resources from Department of Anesthesiology, University of Wuerzburg, Grant 1518 TG 72, Josef-Schneider Str. 2, D-97080 Germany; Helsinki University Central Hospital, HUS-EVO Grant TYH 0324, University of Helsinki, Finland; AstraZeneca, Tinsdaler Weg 183, D-22876 Wedel, Germany; and GlaxoSmithkline, Alsterufer 1, D-20354 Hamburg, Germany. The authors would like to thank the reviewers and the editors for a number of helpful comments that led to significant improvements in the manuscript.

\section{References}

Apfel, C. C., Korttila, K., Abdalla, M., Biedler, A., Kranke, P., Pocock, S. J. and Roewer, N. (2003). An international multicenter protocol to assess the single and combined benefits of antiemetic interventions in a controlled clinical trial of a $2 \times 2 \times 2 \times 2 \times 2 \times 2$ factorial design (IMPACT). Control Clin Trials 24, 736-751.

Apfel, C. C., Korttila, K., Abdalla, M., Kerger, H., Turan, A., Vedder, I., Zernak, C., Danner, K., Jokela, R., Pocock, S. J., Trenkler, S., Kredel, M., Biedler, A., Sessle, D. I., Roewer, N. and Investigators, I. M. P. A. C. T. (2004). A factorial trial of six interventions for the prevention of postoperative nausea and vomiting, $N$ Engl $J$ Med 350, 2441-2451.

Blizzard, L. and Hosmer, D. W. (2006). Parameter estimation and goodness-of-fit in $\log$ binomial regression. Biom J. 48, 5-22.

Carter, R. E., Lipsitz, S. R. and Tilley, B. C. (2005). Quasi-likelihood estimation for relative risk regression models. Biostatistics 6 , 39-44.

Cornfield, J. (1951). A method of estimating comparative rates from clinical data: applications to cancer of the lung, breast, and cervix. J. Natl Cancer Inst 11, 1269-1275.

Grizzle, J. E., Starmer, C. F. and Koch, G. G. (1969). Analysis of categorical data by linear models. Biometrics 25, 489-504.

Lu, M., Tilley, B. C. and t PA Stroke Trial Study Group, N. I. N. D. S. (2001). Use of odds ratio or relative risk to measure a treatment effect in clinical trials with multiple correlated binary outcomes: data from the ninds t-pa stroke trial. Stat Med 20, 1891-1901. 
SAS Institute Inc. (2001). SAS/STAT User's Guide. SAS Institute Inc.

Spiegelman, D. and Hertzmark, E. (2005). Easy SAS calculations for risk or prevalence ratios and differences. Am J Epidemiol 162, 199-200.

Wacholder, S. (1986). Binomial regression in GLIM: estimating risk ratios and risk differences. Am J Epidemiol 123, 174-184.

Zeger, S. L. and Liang, K. Y. (1986). Longitudinal data analysis for discrete and continuous outcomes. Biometrics 42, 121-130.

Zhang, J. and Yu, K. F. (1998). What's the relative risk? A method of correcting the odds ratio in cohort studies of common outcomes. JAMA 280, 1690-1691.

Zou, G. (2004). A modified poisson regression approach to prospective studies with binary data. Am J Epidemiol 159, 702-706.

Received October 3, 2007; accepted November 1, 2007.

Rickey E. Carter

Division of Biomedical Statistics \& Informatics

Health Sciences Research

200 First Street SW

Rochester, MN 55905 USA

Carter.rickey@mayo.edu

Xuyang Zhang

Nationwide Insurance

zhangx5@Nationwide.com

Robert F. Woolson

Department of Biostatistics, Bioinformatics and Epidemiology

Medical University of South Carolina

Charleston, SC 29425, USA

woolson@musc.edu

Christian C. Apfel

Perioperative Clinical Research Core

University of California San Francisco

UCSF Medical Center at Mt Zion

1600 Divisadero, C-447

San Francisco, CA 94143

apfelc@anesthesia.ucsf.edu 